\title{
Comparison of absorbing boundary conditions and numerical modeling of electromagnetic wave for multi component GPR
}

\author{
Yoshinori Sanada \\ Dept. of Earth Resources Engineering, Kyoto University, Kyoto, 606-8501, Japan. \\ e-mail: sanada@tansa.kumst.kyoto-u.ac.jp
}

\begin{abstract}
:
In this paper, numerical modeling of electromagnetic (EM) wave for multi component GPR is developed. For numerical modeling, the FDTD (Finite Difference Time Domain) method is used. In the accurate wave propagation modeling, efficient absorbing boundary condition is very important. The perfect matched layer (PML) and Cerjan's absorbing condition is compared. The result shows PML is more effective than Cerjan's one. The EM reflected waves are simulated with two different antenna arrays with varying structure. The results indicate that multi component GPR obtains more information than conventional one component GPR.
\end{abstract}

\section{Introduction}

Applications of GPR expands more and more, for example civil and environmental engineering (Davis and Annan, 1989), archaeological survey (Goodman, 1994) and etc. Usually survey lines are located cross the structures and antenna is cross the survey line. However in most of cases it is difficult to know the structure previously. The most of antenna of GPR are bow-tie antenna. It is known well that EM waves has polametric characters. The multi component GPR will overcome the problem and the information from multi component antenna expects to help to estimation subsurface more detail. In this paper we discussed the multi component GPR using numerical examples.

First the absorbing boundary condition is discussed. In this paper the FDTD method (Yee, 1966 ) is used for the numerical simulation. The FDTD method, which solves Maxwell's equations with explicit finite difference method on the Staggerd grid, was developed by Yee in 1966. The method is very speedy and powerful for solving electromagnetic (EM) phenomena such as EM wave propagation. In Geophysical exploration field, Bergmann, et al. (1998), Wang and Tripp (1996) and many people have used it. To improve the efficiency of calculation, good absorbing boundary condition is required. Mur (1980) used the one way wave equation and Cerjan (1985) used the attenuation material around the calculation area. And Berenger (1998) developed the PML method and reduced reflections successfully. Here we compared PML and Cerjan's absorbing boundary condition.

Secondaly the observed wave forms and difference from different component of antenna are discussed.

\section{Absorbing Boundary Condition}




\subsection{Perfect Matched Layer}

First, assuming plane EM wave incidents vertically from vacuum to magnetic attenuation media. When the impedance matching condition, wave impedance in the vacuum is equivalent with that in the media, is met, the reflection coefficient equals zero independent with frequencies. In other word the EM wave incident without any reflection. However if the EM wave incident with angles, the reflection coefficient is not zero only the condition. To overcome the condition, new conductivity and permeability are installed. The calculation area is covers around by the nonphysical material which meets the condition that EM wave incident with any angle. This condition is called PML absorbing boundary condition.

Here we assume TM wave that has only $\mathrm{z}$ - electric field. The wave that travels in $\mathrm{x}$ direction and the wave in $\mathrm{z}$ direction are separated. Then the impedance matching should meat for normal incident wave.

In order to install the PML condition into FDTD method, the width of absorbing boundary should be finite width. To prevent unnecessary reflection from outside boundary, the attenuation in the absorbing boundary zone must be gradually decreasing and the outer boundary is able to surrounded by perfect electric walls or perfect magnetic walls. The maximum conductivity, $\sigma_{\max }$ is defined as following equation.

$\sigma_{\max }=-\frac{(M+1) \varepsilon_{0} c}{2 L \Delta x} \ln |R(0)|$

Where, $\mathrm{M}$ is constant giving conductivity distribution, $|R(0)|$ is desired reflection coefficient, $\mathrm{L}$ is the number of layers, $\varepsilon_{0}$ is permittivity of vacuum, $\mathrm{C}$ is the velocity of light and $\Delta x$ is cell size.

\subsection{Comparison between PML and Cerjan's method}

Fig. 1 shows the electric wave forms reflected from the absorbing boundary in 2-dimensional model. The source is located in the center of calculation area. The left are reflections from PML with 4, 8 and 12 layers, respectively. The right are ones from Cerjan's boundary with 20,40 and 80 layers. While the reflections from PML with 8 and 12 layers are absorbing well, Cerjan's method need more 20 layers. The calculation times in the cases are shown in table 1. PML has good performances in the total efficiency. The efficiency would be more remarkable in bigger or 3-dimensional case. 
Table 1 Comparison of calculation condition and calculation time between PML and Cerjan.

\begin{tabular}{|l|c|l|c|}
\hline $\begin{array}{l}\text { Absorbing } \\
\text { Boundary } \\
\text { Condition }\end{array}$ & $\begin{array}{l}\text { Boundary } \\
\text { width }\end{array}$ & $\begin{array}{l}\text { Computational } \\
\text { region }\end{array}$ & $\begin{array}{l}\text { Calculation } \\
\text { time }(\mathrm{sec})\end{array}$ \\
\hline PML & 4 & $208 \times 208$ & 12 \\
\hline PML & 6 & $216 \times 216$ & 14 \\
\hline PML & 20 & $240 \times 240$ & 17 \\
\hline Cerjan & 20 & $240 \times 240$ & 22 \\
\hline Cerjan & 40 & $280 \times 280$ & 32 \\
\hline Cerjan & 80 & $360 \times 360$ & 59 \\
\hline
\end{tabular}

\section{Multi-component GPR}

To show the difference by antenna direction, the EM wave forms are calculated in the different angle of step model shown in Fig. 2. The survey line is on the surface in the center of the models and above the steps with $90,60,45,30$ degree The wave forms recorded with parallel to the survey line are shown in Fig. 3. The less angle, parallel to survey line, is, the more horizontally the reflections are. However it is difficult to estimate the position of the step subsurface. The wave forms recorded with cross to the survey line are shown in Fig. 4. Similar to Fig.3, the less angle, parallel to survey line, is, the more horizontally the reflections are. However the reflections curvatures are much different from Fig. 3. The reason comes from difference of radiation patterns by antenna direction. This results indicate the possibility to distinguish the angles of step structure.

\section{Conclusions}

In this paper we discussed multi component GPR. First, to improve the efficiency of calculation, the evaluation of PML and Cerjan's absorbing boundary conditions. The results shows PML is much efficient for the dimensions of calculation scales and reduces calculation time. The efficiency would be more remarkable in bigger or 3-dimensional case. Secondary, The observed wave forms and difference from different component of antenna are discussed. the difference between the component comes from radiation pattern of the antenna. This results indicate the possibility to distinguish the angles of step structure. These numerical results indicate multi component GPR provide us much more information than single component GPR.

In the future works, more detail and quantitive analysis of polametric radiation and develop imaging method using multi component GPR.

Acknowledgement: The author thanks to Akihiko Mouri and Takuya Nishiuchi for helping calculations. 


\section{References}

Bergmann, T., Robertsson, J. O. A. and Holliger, K., 1998, Finite-difference modeling of electromagnetic wave propagation in dispersive and attenuating media: Geophysics, Soc. of Expl. Geophys., 63, 856-867.

Berenger, J.P., 1994, A perfectly matched layer for the absorption of electromagnetic waves, Journal of Computational Physics, $114,1,185-200$.

Cerjan, C., Kosloff, D., Kosloff, R. and Reshef, M., 1985, A nonreflecting boundary condition for discrete acoustic-wave and elastic-wave equations (short note): Geophysics, Soc. of Expl. Geophys., 50, 705-708.

Davis, J. L., and Annan, A. P., 1989, Ground-penetrating radar for high-resolution mapping of soil and stratigraphy: Geophys. Prosp.,37, 531-551.

Goodman,D., 1994, Ground-penetrating radar simulation in engineering and archaeology: Geophysics, 59, 224-232.

Mur, G., 1981, Absorbing boundary conditions for finite-difference approximation of the time-domain electromagnetic-field equations: IEEE Trans. Electromagn. Compat., 23, 1073-1077.

Wang, T., and Tripp, A. C., 1996, FDTD simulation of EM wave propagation in 3-D media: Geophysics, $60,110-120$.

Yee, K. S., 1966, Numerical solution of initial boundary value problems involving Maxwell's equations in isotropic media: IEEE Trans. Ant. Propag., 14, 302-307. 


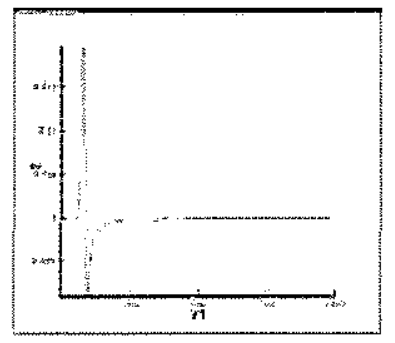

PML4 layers

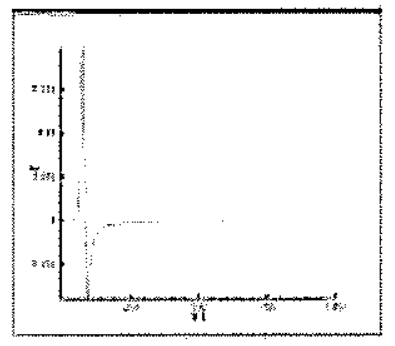

PMI 8 layers

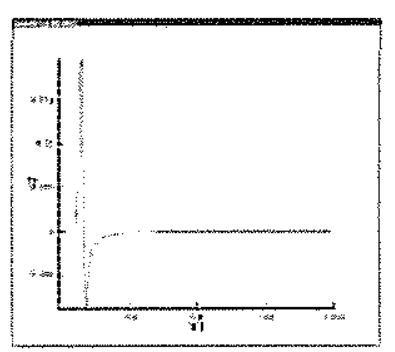

PML12 layers

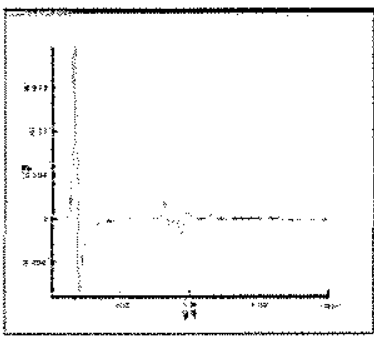

Cerjan20 layers

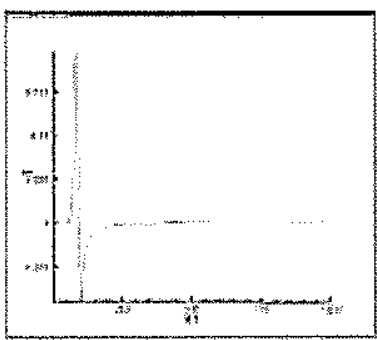

Cerjan 40 layers

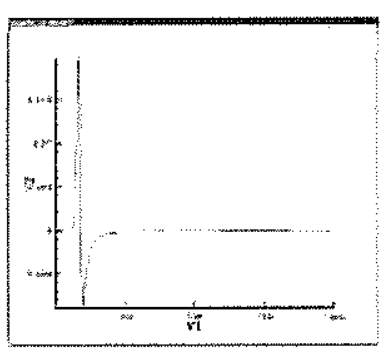

Cerjanso layers

Fig. 1 Comparison of reflection coefficient between PML and Cerjan boundary condition.

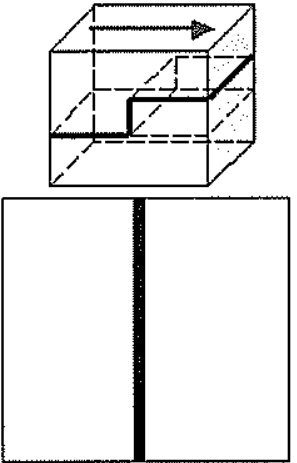

90 degree
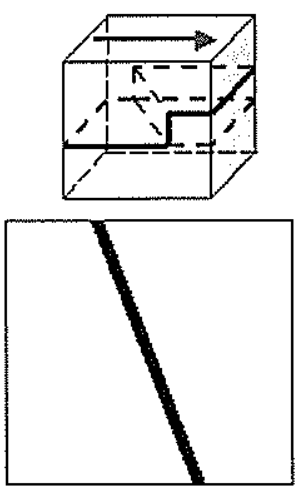

60 degree

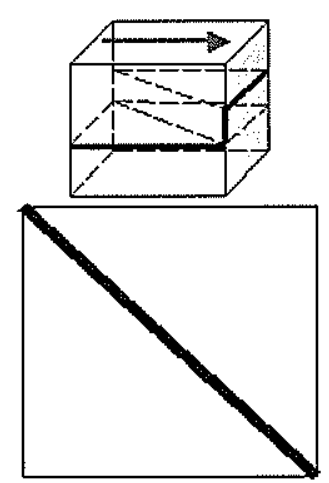

45 degree

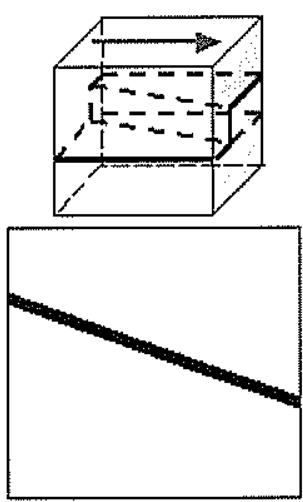

30 degree

Fig. 2 The step models with different angles. The arrows on the surface is survey line and direction. 
survey position

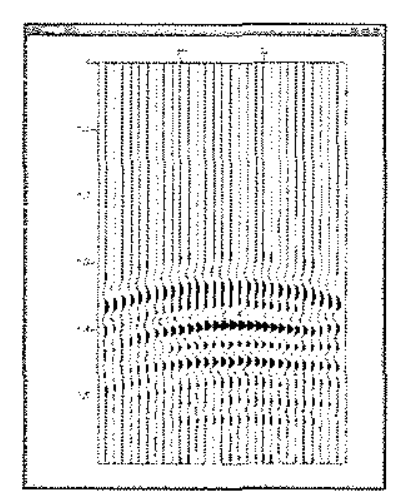

90 degree

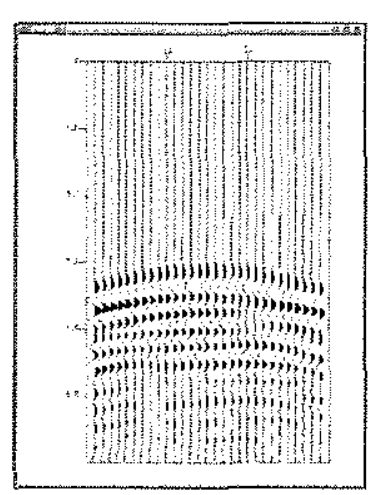

60 degree

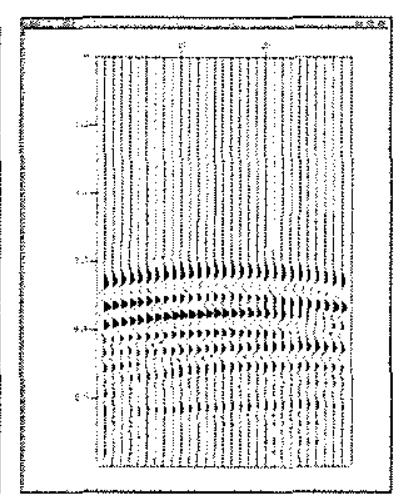

45 degree

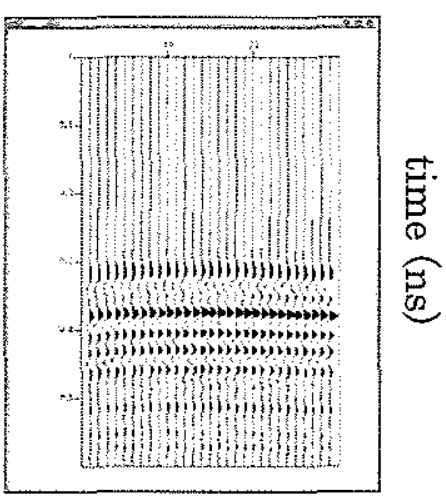

30 degree

Fig. 3 Electric waves at the survey lines. Antenna direction is parallel to survey line.

survey position

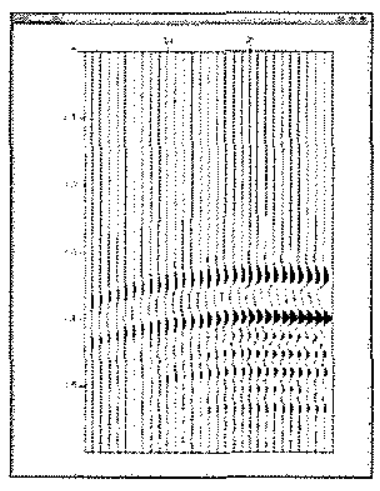

90 degree

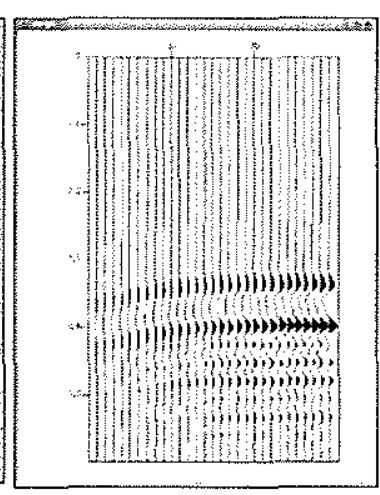

60 degree
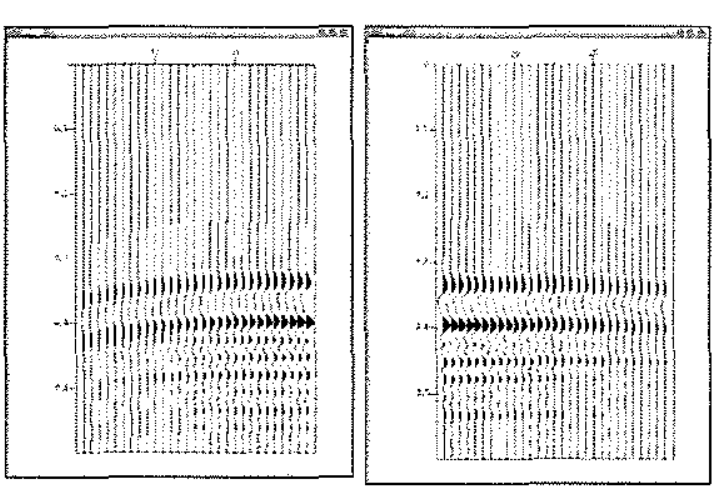

45 degree
点

$$
\text { 急 }
$$

